Bio - grafia. Escritos sobre la Biología y su Enseñanza. ISSN 2027

Edición Extraordinaria. p.p. 1191- 1203

Memorias del VIII Encuentro Nacional de Experiencias en Enseñanza de la Biología y la Educación Ambiental. III Congreso Nacional de Investigación en Enseñanza de la Biología.

\title{
REPRESENTACIONES SOCIALES DE AMBIENTE Y EDUCACIÓN AMBIENTAL EN DOCENTES DE BÁSICA SECUNDARIA Y MEDIA
}

\section{SOCIAL REPRESENTATIONS OF AMBIENCE AND ENVIRONMENTAL EDUCATION IN TEACHERS OF BASIC SECONDARY AND AVERAGE}

\section{Por: José Fernando Ramírez Bermúdez $z^{1}$}

\section{RESUMEN}

Posicionar la educación ambiental en el sector educativo, se ha convertido en uno de los grandes retos de la educación en el país. Al indagar las representaciones sociales de ambiente y educación ambiental en docentes se establece un campo de reflexión y discusión sobre la forma como se están educando y como fueron educados los profesionales que orientan la educación ambiental en el sector escolar. Aunque se han generados políticas nacionales $e$ internacionales sobre formación de ciudadanos comprometidos con el ambiente, aún no es evidente una metodología clara sobre la investigación en educación ambiental en la escuela.

Esta investigación tiene como objeto hacer un diagnóstico educativo-ambiental a partir de la búsqueda y análisis de las representaciones sociales de ambiente y educación ambiental que tienen los docentes de básica secundaria y media en la provincia de Vélez Santander, específicamente en el municipio de Chipatá.

Bajo esta premisa, este trabajo presenta la diversidad de representaciones sociales y las visiones compartidas relacionadas con el ambiente y la educación ambiental, así como sus actitudes, prácticas ambientales y propuestas, en los que se aprecian sus posturas epistemológicas, pedagógicas, éticas, políticas y ambientales, revelando la importancia del cuidado del ambiente en una comunidad educativa. De igual forma, se comprenderá el rol

\footnotetext{
${ }^{1}$ Licenciado en Ciencias Naturales y Educación Ambiental. Universidad Pedagógica y Tecnológica de Colombia. Jose.ramirez04@uptc.edu.co; jose-fercho@hotmail.com
} 
Bio - grafia. Escritos sobre la Biología y su Enseñanza. ISSN 2027

Edición Extraordinaria. p.p. 1191- 1203

Memorias del VIII Encuentro Nacional de Experiencias en Enseñanza de la Biología y la Educación Ambiental. III Congreso Nacional de Investigación en Enseñanza de la Biología.

social del profesorado y su incidencia en la construcción de significados acerca de las realidades ambientales en los contextos locales y regionales.

PALABRAS CLAVE: REPRESENTACIONES SOCIALES, AMBIENTE, EDUCACIÓN AMBIENTAL, DOCENTES.

\begin{abstract}
Position the environmental education in the educational sector, has become one of the major challenges of education in the country. On having investigated the social representations of ambience and environmental education in teachers, there is established a field of reflection and discussion on the form as they are educated and since there were educated the professionals who face the environmental education in the school. Although there have generated national and international training of citizens committed to the environment policy it is not yet clear clear methodology for research in environmental education at school.
\end{abstract}

This research aims to make an educational-environmental diagnosis from the search and analysis of social representations of environment and environmental education with teachers of primary and middle school in the province of Velez Santander, specifically in the town of Chipata.

Under this premise, this paper present the diversity of social representations and shared visions related to the environment and environmental education as well as their attitudes, environmental practices and proposals, which epistemological, pedagogical, ethical, political positions are appreciated and environmental, revealing the importance of protecting the environment in an educational community. Similarly, you will understand the social role of the faculty and its incidence in the construction of meanings about the environmental realities in local and regional contexts.

KEY WORDS: SOCIAL REPRESENTATIONS, AMBIENCE, ENVIRONMENTAL EDUCATION, TEACHERS. 
Bio - grafia. Escritos sobre la Biología y su Enseñanza. ISSN 2027

Edición Extraordinaria. p.p. 1191- 1203

Memorias del VIII Encuentro Nacional de Experiencias en Enseñanza de la Biología y la Educación Ambiental. III Congreso Nacional de Investigación en Enseñanza de la Biología.

\section{INTRODUCCIÓN}

Somos constructores de significados que compartimos socialmente (Pozo, 1998), permanentemente utilizamos las creencias, teorías y explicaciones que elaboramos sobre el mundo para comprender la realidad, reducir la incertidumbre frente a los problemas y adelantar acciones que materialicen nuestros pensamientos.

Reconocer las representaciones sociales de ambiente y educación ambiental (EA) se puede constituir en un referente para diseñar estrategias que permitan aportar a las prácticas, currículos y demás procesos pedagógicos, de las diferentes áreas del conocimiento en la escuela. Esto se ratifica en la Política Nacional de Educación Ambiental, de los ministerios de Educación Nacional y del Medio Ambiente (2002), al establecer como prioridad que las instituciones educativas deben desarrollar estrategias tendientes a introducir la dimensión ambiental como componente importante de las diferentes áreas del currículo escolar. En consecuencia, es preciso señalar que esta investigación pretende aportar a la formación de un pensamiento y una cultura ambiental acorde con los propósitos misionales y al desarrollo de la Política Ambiental educativa del país.

El municipio de Chipatá, viene adelantando procesos de dinámicos que buscan la incorporación de la dimensión ambiental en todos los sectores que lo componen especialmente en el sector educativo. Bajo este perspectiva, Gutiérrez y Pozo (2006), afirman que en el sector educativo-ambiental existe la limitación de no contar con una formación rigurosa, hecho que se ratifica con la poca institucionalización del saber ambiental en aspectos académicos, administrativos y de gestión. El estudio de las representaciones sociales avanza en dos perspectivas, la primera de ellas radica en la posibilidad de reconocer las ideas, los valores y las actitudes relacionados con el ambiente y educación ambiental, por parte de los docentes, así como las formas de apropiar, simbolizar y significar un escenario común como es las instituciones educativas del municipio, con diferentes capas de interacciones, donde se presentan convergencias, tensiones y problemáticas que emergen y otras que se acentúan de acuerdo con lo suscitado por los docentes; aportes que sin duda pueden servir de base para ser considerados en los procesos de reflexión que contribuyan a la generación de un pensamiento y cultura 
Bio - grafia. Escritos sobre la Biología y su Enseñanza. ISSN 2027

Edición Extraordinaria. p.p. 1191- 1203

Memorias del VIII Encuentro Nacional de Experiencias en Enseñanza de la Biología y la Educación Ambiental. III Congreso Nacional de Investigación en Enseñanza de la Biología.

ambientales que a futuro impactarán los diferentes escenarios que componen el municipio y sus centros educativos. Perez, P., \& Guzman (2013).

\section{Representaciones Sociales}

Las representaciones sociales (RS) constituyen una categoría compleja de análisis, en la cual confluyen diversos marcos interpretativos de la realidad, con los cuales las personas evalúan y construyen explicaciones, producto de los procesos comunicativos y la interacción social (Araya, 2002). Por estas razones las representaciones sociales revalidan la construcción social del saber, con el cual la gente se desenvuelve, organiza su vida y toma decisiones. Según Jodelet (1984) dicho saber espontáneo e ingenuo se denomina sentido común, como una forma de concebir la realidad, de dotar de sentido las prácticas, de orientar los comportamientos y promover las relaciones interpersonales. Este conocimiento se construye colectivamente a partir de los intercambios, las experiencias y las interacciones con otras personas, y a través de las creencias y valores que circulan en instituciones educativas y culturales, Internet, televisión, al igual que en las esferas pública y privada (Terrón y González, 2009) que permiten configurar las representaciones y compartir significados dada su connotación social.

Los primeros estudios de las representaciones sociales se remontan a los trabajos de Moscovici, en los años sesenta, quien acuñó el concepto en su famosa tesis doctoral de 1961, El psicoanálisis: su imagen y su público. En este y posteriores documentos, Moscovici propone como objeto de estudio el conocimiento de sentido común, desde una perspectiva psicológica y como forma de construcción colectiva de la realidad entendiendo la representación como: una modalidad particular del conocimiento, cuya función es la elaboración de los comportamientos y la comunicación entre los individuos [...] La representación es un corpus organizado de conocimientos y una de las actividades psíquicas gracias a las cuales los hombres hacen inteligible la realidad física y social, se integran en un grupo o en una relación cotidiana de intercambios, liberan los poderes de su imaginación (Moscovici, 1979). Consecuentemente, Jodelet (1984), profundiza el análisis del campo de representación asociado al conocimiento de sentido común, articulando procesos intelectuales y sociales, en lo que se asocia con una forma de pensamiento social: Este conocimiento (espontáneo, ingenuo) se constituye a partir de nuestras experiencias, pero también de las informaciones, conocimientos y modelos de pensamiento que recibimos y 
Bio - grafia. Escritos sobre la Biología y su Enseñanza. ISSN 2027

Edición Extraordinaria. p.p. 1191- 1203

Memorias del VIII Encuentro Nacional de Experiencias en Enseñanza de la Biología y la Educación Ambiental. III Congreso Nacional de Investigación en Enseñanza de la Biología.

transmitimos a través de la tradición, la educación y la comunicación social. De este modo, ese conocimiento es en muchos aspectos un conocimiento socialmente elaborado y compartido.

Las representaciones sociales cuentan con una estructura que comprende tres dimensiones (información, campo de representación y actitud), que son socialmente compartidas por un grupo como una secuencia de elaboraciones en el tiempo con un carácter dinámico y que les permite interpretar la realidad (Terrón y González, 2009). Estas tres dimensiones se entienden como:

- Información: conjunto de conocimientos que poseen los sujetos sobre un objeto social.

- Campo de representación: unidad jerarquizada de proposiciones, opiniones y evaluaciones, así como sus diferencias contenidas en la RS; se organiza en torno al núcleo figurativo y revela la situación de la práctica del objeto representado.

- Actitud, postura ante el objeto: expresa los componentes afectivos favorables o desfavorables hacia el objeto de representación. Estos referentes teórico-conceptuales de las representaciones sociales cobran especial interés en la investigación educativa, al comprender la forma en que las personas desde edades tempranas conciben el mundo y cómo estas representaciones se configuran e inciden en su producción cognitiva, y a su vez cómo las instituciones educativas aportan en la construcción de referentes simbólicos, pautas de comportamiento, organización del conocimiento y en las formas de pensar y construir la realidad (Moscovici, 1979).

Dada la relevancia que tiene la educación y el rol de los maestros en el acto de educar, es de nuestro interés poder identificar las representaciones sociales de los docentes en formación, quienes son ciudadanos, estudiantes y a su vez se preparan para ser futuros maestros, al formar parte de una institución universitaria formadora de formadores, que facilita el intercambio de conocimientos, el fomento de valores, el encuentro de visiones, creencias, prácticas y formas de significar la realidad.

\section{AMBIENTE.}

Leff (2006) plantea que la epistemología ambiental es una aventura del conocimiento que busca un horizonte del saber. Esta idea invita a reflexionar sobre cual es el nicho del ambiente para entender procesos de acción y reflexión en un contexto. El ambiente no 
Bio - grafia. Escritos sobre la Biología y su Enseñanza. ISSN 2027

Edición Extraordinaria. p.p. 1191- 1203

Memorias del VIII Encuentro Nacional de Experiencias en Enseñanza de la Biología y la Educación Ambiental. III Congreso Nacional de Investigación en Enseñanza de la Biología.

resulta ser un concepto fácil de definir porque sobre el se tejen diversas interpretaciones que dependiendo dl campo de conocimiento donde de vea adquiere diferentes representación y significados de ver y actuar en el mundo. Es por esto que en este trabajo tomaremos el ambiente como un saber que el principal objeto es la reflexión, que nace de la critica y abre caminos para encontrar nuevos caminos de conocimiento de racionalidad científica, social y compleja.

Definido el concepto de ambiente es indispensable concebir el concepto de educación ambiental.

\section{Educación Ambiental}

La educación ambiental es una corriente de pensamiento y acción de alcance internacional que adquiere gran relevancia en la década de los 70 tras la necesidad de combatir la degradación del ambiente a nivel mundial.

Es así como el término de educación ambiental fue utilizado por primera vez en Estocolmo en la conferencia internacional del medio ambiente. En Colombia, el Ministerio de Educación y de Medio Ambiente en el año 2002 crean la Política Nacional de Educación Ambiental, la cual establece que la educación ambiental debe ser considerada como un proceso que permite al individuo comprender las relaciones de interdependencia con su entorno, a partir del conocimiento reflexivo y crítico de la realidad biofísica, social, política, económica y cultural para que a partir de la apropiación de la realidad concreta se pueda generar en el y en su comunidad actitudes de valoración y respeto por el ambiente.

\section{METODOLOGÍA}

El presente estudio se enmarca desde un paradigma interpretativo cuyo interés es el estudio de los significados e intenciones de las acciones humanas desde la perspectiva de los agentes sociales. Igualmente el enfoque investigativo es el cualitativo dado que busca identificar, describir, analizar e interpretar un fenómeno, dado que hace de la cultura su concepto central. Por tanto y desde este enfoque se espera estudiar los significados de las acciones humanas y de la vida social (Pantoja, 2009). 
Bio - grafia. Escritos sobre la Biología y su Enseñanza. ISSN 2027

Edición Extraordinaria. p.p. 1191- 1203

Memorias del VIII Encuentro Nacional de Experiencias en Enseñanza de la Biología y la Educación Ambiental. III Congreso Nacional de Investigación en Enseñanza de la Biología.

El método propuesto para este ejercicio investigativo y desde el paradigma interpretativo, es el estudio de caso, como una estrategia que en palabras de Merriam (1988) presenta cuatro propiedades esenciales: particularista, descriptivo, heurístico e inductivo. Estas propiedades permiten realizar un acercamiento reflexivo a una realidad en particular, en este caso a las representaciones sociales de ambiente y educación ambiental de docentes en el municipio de Chipatá Santander.

\section{Población.}

La población participante en esta investigación serán docentes de básica secundaria y media del municipio de Chipatá Santander. Por ser este un estudio cualitativo y basado en el método de estudio de casos, por conveniencia investigativa se tomara una muestra del universo a estudiar, pero es los resultados obtenidos no se podrán generalizar para toda la población.

Para la recolección de información se implantará la entrevista a profundidad, la cual será validada por expertos (3), los cuales tienen como características (2) dominio de la temática en ambiente y educación Ambiental y (1) neutral que no tiene relación la problemática pero si con la forma de realizar las preguntas y su respectiva intencionalidad, a través de esta entrevista se buscará entrar en la vida del otro, penetrar y detallar en lo trascendente, descifrar y comprender los gustos, los miedos, las satisfacciones, las angustias, zozobras y alegrías, significativas y relevantes del entrevistado; consiste en construir paso a paso y minuciosamente la experiencia del otro. La entrevista en profundidad sigue el modelo de plática entre iguales, "encuentros reiterados cara a cara entre el investigador y los informantes" (Taylor y Bogdan, 1990) reuniones orientadas hacia la comprensión de las perspectivas que tienen los informantes respecto de sus vidas, experiencias o situaciones, tal como las expresan con sus propias palabras. De igual forma, para dar mayor solidez a la investigación se realizará un grupo focal que será integrado por máximo 4 docentes que se escogerán de manera aleatoria o por conveniencia investigativa. El objetivo del grupo focal es discutir y reflexionar desde las experiencias personales los hechos sociales, naturales y culturales que afectan el ambiente y su forma de educar. 
Bio - grafia. Escritos sobre la Biología y su Enseñanza. ISSN 2027

Edición Extraordinaria. p.p. 1191- 1203

Memorias del VIII Encuentro Nacional de Experiencias en Enseñanza de la Biología y la Educación Ambiental. III Congreso Nacional de Investigación en Enseñanza de la Biología.

\section{Fases de la investigación}

La presente investigación adoptará los principios teóricos propios de la metodología cualitativa de tipo descriptivo-interpretativo, con la que se pretenden reconocer las representaciones sociales de ambiente y educación ambiental en docentes de básica secundaria y media. Es de anotar, cómo en los enfoques metodológicos cualitativos de investigación la validez de la interpretación reside en el acercamiento y la comprensión desde el interior de las situaciones que se estudian, privilegiando la dimensión subjetiva de la realidad y promoviendo el diálogo de saberes (Torres, 1996), lo cual permite que en los contextos sociales sus miembros interactúen compartiendo el significado y el conocimiento que tienen de sí mismos y de su realidad.

La presente investigación se realizará en tres fases:

1. Documentación: en esta fase el grupo de investigadores realizó una revisión minuciosa sobre las principales tendencias sobre investigaciones en representaciones sociales. En ella, la información recolectada se categorizo para realizar la identificación, caracterización y análisis de las representaciones sociales de los docentes.

2. Inmersión en el contexto y recolección de la información: durante esta fase se aplicó los diferentes instrumentos (entrevista a profundidad y grupo focal) diseñados y se recolectó, identificó y caracterizó las representaciones sociales de ambiente y educación ambiental de docentes de básica secundaria y media del municipio de Chipatá.

3. Análisis y construcción de conocimiento: Durante esta fase, se analizará las representaciones sociales de los docentes, a través de categorías emergentes del mismo discurso de ellos, se categorizaran con respecto a una matriz ya establecida y finalmente se dará cumplimiento al objetivo de la investigación.

\section{RESULTADOS Y DISCUSIÓN}

En primera instancia se debe resaltar que esta investigación está en desarrollo, por tal motivo se relacionan algunos resultados producto de las actividades realizadas hasta el momento. 
Bio - grafia. Escritos sobre la Biología y su Enseñanza. ISSN 2027

Edición Extraordinaria. p.p. 1191- 1203

Memorias del VIII Encuentro Nacional de Experiencias en Enseñanza de la Biología y la Educación Ambiental. III Congreso Nacional de Investigación en Enseñanza de la Biología.

Teniendo como base las fases metodologías de la investigación, la parte documental se constituye como uno de los ejes fundamentales en este proceso reflexivo y generador de conocimiento. En primer lugar, la provincia de Vélez especialmente el municipio de Chipatá Santander se encuentra ubicada la institución educativa (IE) de Tierra negra, la cual es una IE de carácter oficial y de género mixto, atiende a la población escolar en los niveles de Preescolar y Básica. Para el 2013 registra en el Sistema Integrado de Matricula SIMAT, 106 estudiantes provenientes de veredas del municipio de Chipatá: Mirabuenos, Centro, Toroba Ropero, y las aledañas que pertenecen al municipio de Vélez: Loma Alta y Guayabal. Su planta docente la conforman 22 profesionales en el área de la educación y otras especialidades. La Misión que persigue la IE es la formación integral de niños y jóvenes, fundamentada en los valores humanos, fortaleciendo el desarrollo de actitudes críticas, reflexivas, democráticas y ambientales, que potencien la formación de líderes constructores de una sociedad comprometida con el desarrollo social, económico, cultural, ambiental y ético de la comunidad, la región y el país. La EA se referencia desde los contenidos del plan de estudio de Ciencias Naturales, y el PRAE se contempla en el PEI de manera activista, caracterizado por el desarrollo de acciones de reciclaje de basuras y jornadas anuales de instalación de jardines.

En segundo lugar, se esta realizando un Cartografía Informacional Bibliográfico (CIB) mediante el cual se construye una opción para orientar búsquedas, para seleccionar fuentes bibliográficas y determinar el desarrollo conceptual de perspectivas de investigación (ANDRÉ, 2009; CREMADES, 2011; MOLINA, 2012). André (2009), establece la necesidad del desarrollo de una competencia informacional, en la que se trata de mapear los contenidos más relevantes de una obra científica, filosófica, literaria. Medeiros (1999), apuntado por André (2009: 63), anota que (...) "mapear un contenido significa sintetizarlo, lo que requiere una lectura atenta de las informaciones, su comprensión, la identificación de las ideas principales del autor y su registro escrito de modo conciso, coherente y objetivo"

En concordancia con lo anterior se relaciona la forma como se hace la respectiva sistematización de los documentos analizados para el desarrollo de la perspectiva investigativa en torno a las representaciones sociales de ambiente y educación ambiental. 
Memorias del VIII Encuentro Nacional de Experiencias en Enseñanza de la Biología y la Educación Ambiental. III Congreso Nacional de Investigación en Enseñanza de la Biología.

Tabla 1. Cartografía Informacional Bibliográfico sobre Representaciones Sociales

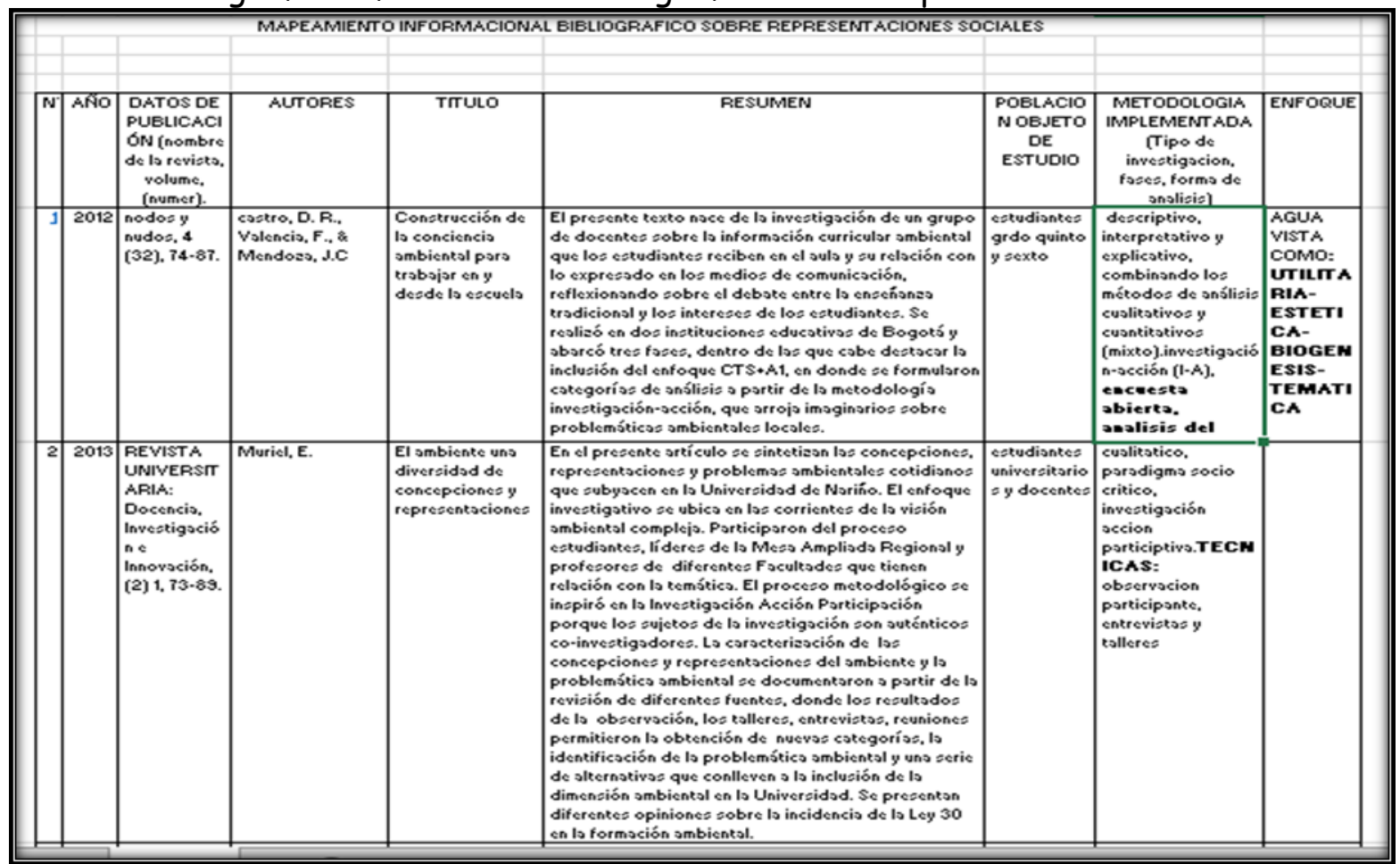

Fuente: del autor

El CIB permite evidenciar las maneras de como se ha construido la parte conceptual y metodológica en torno a las representaciones sociales. Es así, es de resaltar que las metodologías implementadas tienen un corte de carácter interpretativo, con uso de técnicas de recolección de información como las encuestas, entrevistas y talleres. En cuanto a los enfoques encontrados, se ve al ambiente como un recurso, un sitio, ecologista y utilitarista, para lo cual se deja de lado la parte cultural y social que de alguna u otra forma hacen parte del ambiente y lo hace más dinámico.

\section{CONCLUSIONES.}

La IE de Tierra Negra aunque cuenta con un PEI bien estructurado dando respuesta a los requerimientos del ministerio de Educación. Sin embargo, no es visible una relación directa con la el desarrollo de la educación ambiental en el plantel. 
Bio - grafia. Escritos sobre la Biología y su Enseñanza. ISSN 2027

Edición Extraordinaria. p.p. 1191- 1203

Memorias del VIII Encuentro Nacional de Experiencias en Enseñanza de la Biología y la Educación Ambiental. III Congreso Nacional de Investigación en Enseñanza de la Biología.

Con respecto, al proceso metodológico basado en el CIB, se puede decir que las categorías Enfoques y metodología implementadas son acertadas para capturar la riqueza del campo conceptual estudiado y que permiten mostrar posibilidades para la investigación del campo en el contexto de las Representaciones Sociales. Bajo esta perspectiva, se encontró que las representaciones sociales de ambiente mas sobresaliente fueron naturalista, globalizante y antropocéntrica, y las representaciones sociales de Educación Ambiental se enmarcan desde las corrientes de Lucié Sauvé como naturalista, conservacionista, resolutiva, práxica y critica

\section{BIBLIOGRAFÍA}

ANDRÉ, C (2011) prática da pesquisa e mapeamento informacional bibliográfico apoiados por recursos tecnológicos: impactos na formação de professores. (Tesis doutoral). Faculdade de Educação Universidade de São Paulo.

Bermúdez, O.; Santos, Z.; Burbano, S.; Mayorga, M.; Castiblanco C., Ripoll, E.; Rico, A.; Herrera, F.; Hurtado, M.; y Mateus, M. (2005). Representaciones Sociales y Mapas Mentales del Campus Universitario. Primera etapa. Facultad de Artes. Instituto de Estudios Ambientales. Universidad Nacional de Colombia. Bogotá: Editorial Antropos.

Gutiérrez, J. \& Pozo, M. (2006). Modelos teóricos contemporáneos y marcos de Fundamentación de la Educación Ambiental. Revista Iberoamericana de Educación, 41, 2168.

Jodelet, D. (1984). La representación social: fenómenos, concepto y teoría. En: Moscovici, S. (ed.). Psicología Social II. Pensamiento y vida social. Psicología social y problemas sociales. Barcelona: Paidós.

Leff (2006). Aventuras de la epistemología ambiental. Mexico. Siglo XXI editores. 
Bio - grafia. Escritos sobre la Biología y su Enseñanza. ISSN 2027

Edición Extraordinaria. p.p. 1191- 1203

Memorias del VIII Encuentro Nacional de Experiencias en Enseñanza de la Biología y la Educación Ambiental. III Congreso Nacional de Investigación en Enseñanza de la Biología.

Merriam, S. (1988). Case study reseach in education a qualitative approach. San Francisco: jossey-Bass.

Ministerio de educación nacional \& ministerio de medio ambiente y desarrollo sostenible.(2002). Política nacional de Educacion ambiental. Bogota. Colombia.

MOLINA, A., MARTÍNEZ, C., A., MOSQUERA, C., J., y MOJICA., L. (2003) Diversidad cultural $e$ implicaciones en la enseñanza de las ciencias: reflexiones y avances. Revista Colombiana de Educación, V. 56, p. 103-128

MOLINA, A.; PÉREZ, M.; CASTAÑO, N.; BUSTOS, E.; SUÁREZ, O.; SÁNCHEZ (2012) M. Mapeamiento informacional bibliográfico en el campo de la enseñanza de las ciencias, contexto y diversidad cultural: el caso del Journal Cultural Studies in Science Education (CSSE). Revista EDUCyT; V. Extra, Diciembre, 2012, p. 197-222.

Moscovici, S. (1979). El psicoanálisis, su imagen y su público. Buenos Aires: Huemal.

Pantoja, A. (2009). Manual Básico para la Realización de Tesinas, Tesis y Trabajos de Investigación. Madrid, España: Editorial EOS.

Perez, M., porras, Y., \& Guzman, H (2013). Representaciones sociales de la educación ambiental y del campus universitario. Una mirada de los docentes en formación de la Universidad Pedagógica Nacional. Revista TED (34). P 47-69.

Pozo, J. I. \& Gómez, M. A. (1998). Aprender y enseñar ciencias. Del conocimiento cotidiano al conocimiento científico. Madrid: Ediciones Morata.

Taylor, S \& Bogdan, R (1990). Introducción a los métodos cualitativos de investigación. Barcelona, Paidós. 
Bio - grafia. Escritos sobre la Biología y su Enseñanza. ISSN 2027

Edición Extraordinaria. p.p. 1191- 1203

Memorias del VIII Encuentro Nacional de Experiencias en Enseñanza de la Biología y la Educación Ambiental. III Congreso Nacional de Investigación en Enseñanza de la Biología.

Terrón, E. y González, E. (2009). Representación y medio ambiente en la educación básica en México. Trayectorias 11 (28), 58-81.

Torres, A. (1996). Estrategias de Investigación Cualitativa. Unidad 1. En: Estrategias y técnicas de investigación cualitativa. Bogotá: 